\section{Multifocal pancreatoblastoma with pseudocyst in a 2-year-old boy}

\section{Introduction}

Pancreatic neoplasms (either endocrine or exocrine) occur rarely in children, causing less than $0.2 \%$ of malignant pediatric deaths. ${ }^{1}$ We report a 2 -year-old boy with a multifocal unresectable pancreatoblastoma and a secondary pseudocyst in the tail. This case highlights the unusual presentation of this rare disease, and its diagnostic and management challenges.

\section{Case report}

A 2-year-old boy was brought to the surgery clinic with progressive painless left upper abdominal distension of 3 months' duration. There was no history of pain, vomiting, fever, or, jaundice. General physical examination was unremarkable. On abdominal examination, an $8 \mathrm{~cm}$ x $10 \mathrm{~cm}$ cystic, non-tender, retroperitoneal swelling was palpable in the left hypochondrium and lumbar region. Ultrasound abdomen was suggestive of a large homogenous hypoechoic lesion in relation to the pancreatic tail, with a bulky pancreatic head. CT abdomen revealed a large, (maximum diameter $\sim 15 \mathrm{~cm}$ ) cystic, homogenously hypodense lesion in the lesser sac arising from the pancreatic tail, with asymmetric contrast enhancement of the medial wall (Figure 1). The pancreatic body and tail were enlarged, and, two more cystic lesions with multiple solid enhancing areas were identified within the head and body. Fat planes with the superior mesenteric vessels were maintained. A radiological diagnosis of multifocal pancreatoblastoma with a secondary pseudocyst was made. Serum alpha fetoprotein levels were raised. Image-guided FNAC from the mass was inconclusive. Total pancreatectomy was planned. On the day of surgery, the child had fever, tachycardia, and tenderness over the lump. At laparotomy, we found a large, infected, cystic lesion in the lesser sac, densely adherent and inseparable from the posterior gastric wall. Due to dense adhesions and poor general condition, curative resection was deferred. External drainage of the infected pseudocyst was performed, with biopsy of the pancreatic head and a thickened area of the of the cyst wall. The patient recovered satisfactorily in the postoperative period.

Histopathological examination revealed small round epitheloid cells with atypical nuclei arranged in uniform nests.

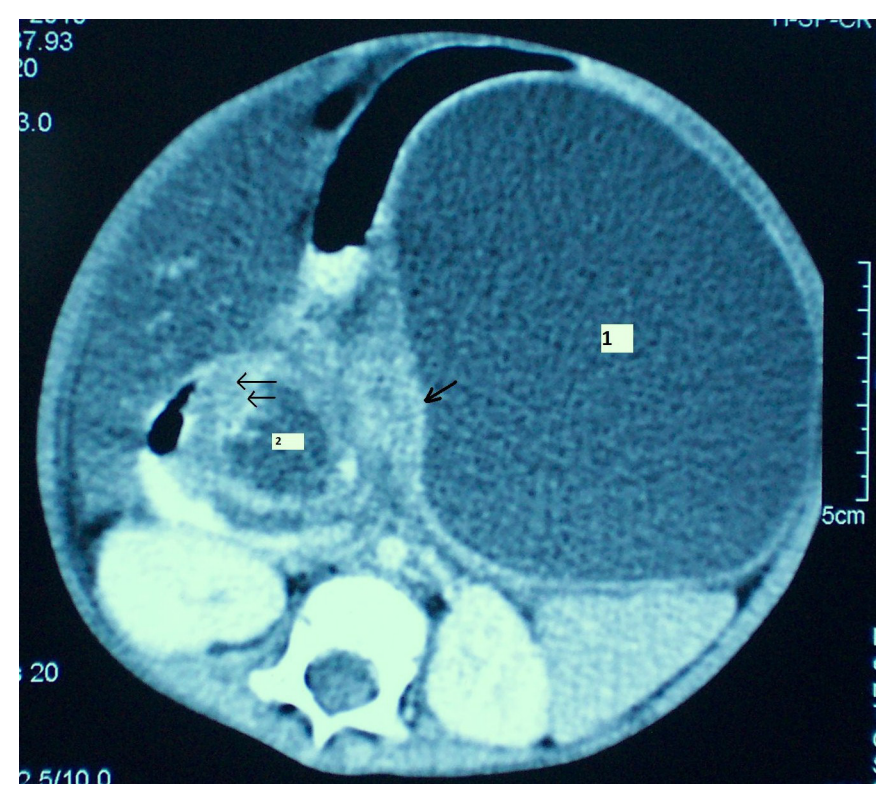

Figure 1: Contrast-enhanced CT scan of a young boy with a large abdominal lump. Single and double arrows represent the solid enhancing areas in the body and head of the pancreas respectively. A large homogenous pseudocyst (marked ' 1 ') is seen in the tail region, and a smaller necrotic area is seen in the head (marked ' 2 '). The entire pancreas has been replaced by the multifocal lesion.

A final diagnosis of multifocal pancreatoblastoma with a secondary pseudocyst was made, and the patient was planned for chemotherapy.

\section{Discussion}

Pancreatoblastoma and solid-papillary epithelial tumor are the most common pancreatic neoplasms in children. Only about 200 cases of pancreatoblastoma have been reported; of those, 60 in English literature. ${ }^{2}$ This contrasts with the situation in adults where pancreatic tumors are more common, and the predominant pathology is adenocarcinoma. The highest incidence is found in the first decade of life with a predilection for males and Asians. The usual presentation is a large, asymptomatic, palpable abdominal mass in a child. Imaging characteristics are a large heterogenous solid-cystic pancreatic lesion, calcifications, and, compression rather than infiltration of surrounding structures. ${ }^{3}$ Elevated serum alpha fetoprotein levels have been reported in upto $68 \%$ of cases. ${ }^{2}$ Though malignant, these tumours have a good prognosis after complete resection. Survival has also been reported in unresectable cases, using a combined-modality approach with surgery, radiotherapy and chemotherapy. ${ }^{4,5}$ These tumors are amenable for various modes of treatment; surgery is the most effective. Complete resection of the tumour offers the best prognosis. 
However, with unresectable or metastatic disease, it is advisable to attempt a trial of chemotherapy. The most frequently used agents are doxorubicin and cisplatinum. ${ }^{5}$

NITIN AGARWAL, MOHAMMAD S FARIDI, PRADEEP SAINI, ARUN GUPTA, NAVNEET KAUR

Correspondence: Dr. Nitin Agarwal Department of Surgery University College of Medical Sciences \& Guru Tegh Bahadur Hospital Delhi 110096, India

Email:_rnitinagarwal76@gmail.com

\section{References}

1. Davey MS, Cohen MD. Imaging of gastrointestinal malignancy in childhood. Radiol Clin North Am 1996;34:717-42.

2. Saif MW. Pancreatoblastoma. JOP. 2007;8:55-63.

3. Chung EM, Travis MD, Conran RM. Pancreatic tumors in children: radiologic-pathologic correlation. Radiographics 2006;26:1211-38.

4. Ohata R, Okazaki T, Ishizaki Y, Fujimura J, Shimizu T, Lane GJ, et al. Pancreaticoduodenectomy for pancreatoblastoma: a case report and literature review. Pediatr Surg Int. 2010;26:447-50.

5. Souzaki R, Tajiri T, Kinoshita Y, Tanaka S, Koga Y, Suminoe A, et al. Successful treatment of advanced pancreatoblastoma by a pylorus-preserving pancreatoduodenectomy after radiation and high-dose chemotherapy. Pediatr Surg Int. 2010;26:1045-8.

\section{Extra-osseous Ewing's sarcoma presenting as obstructive jaundice}

\section{Introduction}

Tefft et al first described a soft tissue tumor in five young children, which was indistinguishable from Ewing's sarcoma (ES) of bone, on light microscopy. ${ }^{1}$ To the best of our knowledge there are less than 10 case reports of intra-abdominal Ewing's sarcoma, and there are no case reports of intra-abdominal Ewing's sarcoma presenting with obstructive jaundice. We therefore report the first case of intra-abdominal Ewing's sarcoma and obstructive jaundice due to compression of the retro-pancreatic bile duct and review the literature. This case also illustrates the difficulties in diagnosis and management.

\section{Case report}

A 50-year-old male patient presented with back pain of 1 month's duration. Abdominal examination revealed a palpable mass located in the lower abdomen. Routine laboratory tests were normal. USG abdomen was performed that showed multiple hypoechoic and heterogenous nodules in the peri-aortic and para-aortic regions. CT abdomen revealed a large lobulated solid mass $(6.8 \mathrm{~cm} \times 9.4 \mathrm{~cm} \times 13.5 \mathrm{~cm})$ in the right lumbar region extending into the right iliac fossa, multiple lymph nodes in the paraaortic, paracaval and retroperitoneal regions. Lymphoma was suspected and FNAC report was inconclusive. Biopsy showed round to polyhedral cells arranged in rosette pattern suggestive of cellular tumor mass and advised histological assessment. After 15 days he again presented to us with jaundice and worsening abdominal distention. Lab reports suggested obstructive jaundice. Repeat CT abdomen (Figure 1) showed large lobulated soft tissue mass $(20 \mathrm{~cm} \times 17 \mathrm{~cm} \times 16$ $\mathrm{cm}$ ) and there was enlargement of all groups of retroperitoneal and mesenteric lymph nodes, which was non-discrete. CBD was dilated $(10 \mathrm{~mm})$ and displaced anteriorly and compressed in its retro pancreatic segment by a large lymph nodal mass. Histology (Figure 2) was suggestive of malignant round cell tumor. Immunohistochemical studies included CD99 (+), synaptophysin ("), and LCA (-). In the context of clinical and

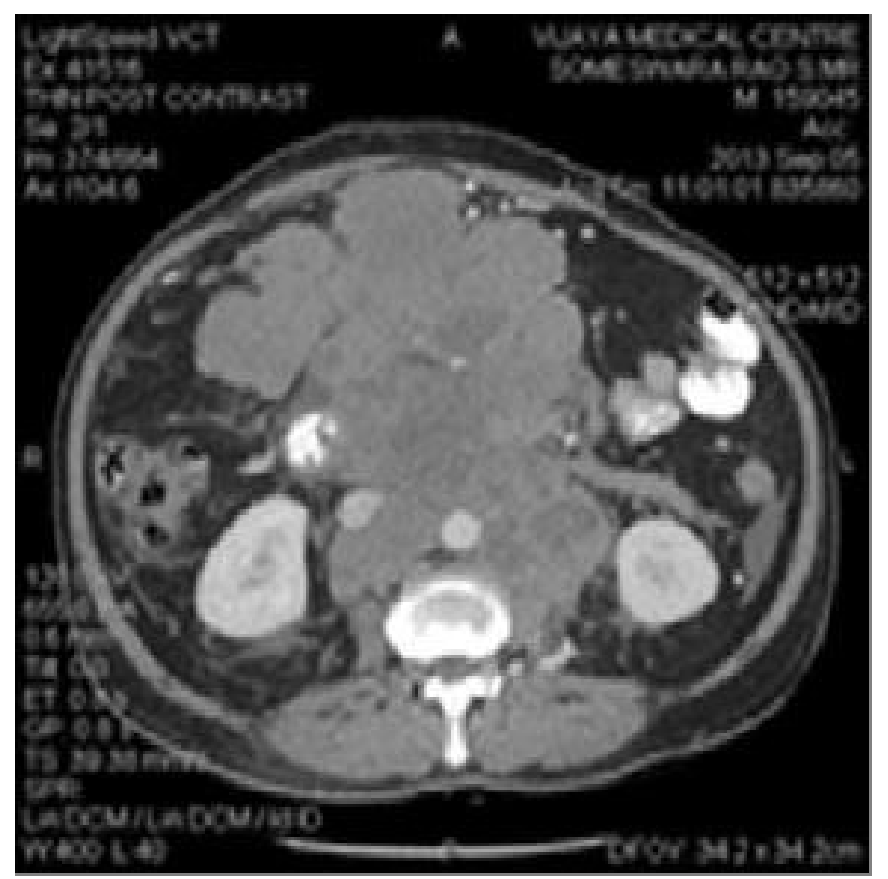

Figure 1: CT scan showing intraabdominal mass with compression of bile duct. 